\title{
Auditing of Discharge Education to Coronary Angiography Patients
}

\author{
Fatemeh Davoodi, ${ }^{1}$ Foroozan Atashzadeh-Shoorideh, ${ }^{2,}$ Hossein Shiri, ${ }^{3}$ Abolfazl Payandeh, ${ }^{4}$ Mahnaz
}

Ilkhani, ${ }^{3}$ Hadi Azimi, ${ }^{5}$ Sara Mahmoody, ${ }^{2}$ and Marjan Mohamadi ${ }^{1}$

${ }^{1}$ Nursing and Midwifery School, Shahid Beheshti University of Medical Sciences, Tehran, Iran

${ }^{2}$ Nursing Management Department, Nursing and Midwifery School, Shahid Beheshti University of Medical Sciences, Tehran, Iran

${ }^{3}$ Department of Medical-Surgical Nursing, Nursing and Midwifery School, Shahid Beheshti University of Medical Sciences, Tehran, Iran

${ }^{4}$ Department of Biostatistics, School of Paramedical Science, Shahid Beheshti University of Medical Sciences, Tehran, Iran

${ }^{5}$ English Language Teaching Department, School of Medicine, Shahid Beheshti University of Medical Sciences, Tehran, Iran

Corresponding author: Foroozan Atashzadeh-Shoorideh, Nursing Management Department, Nursing and Midwifery School, Shahid Beheshti University of Medical Sciences, Tehran, Iran. Tel: +98-2188202519, E-mail: f_atashzadeh@sbmu.ac.ir

Received 2016 August 06; Accepted 2016 August 13.

\begin{abstract}
Background: Coronary angiography (CAG) is one of the procedures for diagnosis of coronary artery disease (CAD). It may have severe complications. Providing patient education based on standards can lead to reduction in complication risks and increase patient satisfaction.

Objectives: Consequently, the present study was conducted to audit discharge education for CAG patients.

Methods: In this descriptive study, 387 discharge educations of CAG patients were observed by event sampling. The data were collected using demographic information of nurses and patients and a checklist formulated on the basis of standards for discharge education of CAG patients. This checklist had 35 items in six dimensions. The validity and reliability of the checklist was examined using content and validity and the Inter-rater correlation coefficient (ICC =97\%). The data was analyzed using SPSS20.

Results: The results showed that the discharge education of CAG patients was weak in all dimensions. The highest education was found for the CAG wound care sub-domain. Education of bleeding control education, nurse-patient communication, changing life style education, emergency events education, and drugs usage dimensions were low.

Conclusions: The findings revealed that common CAG discharge education is not satisfactory in comparison with the standards. Factors that may contribute to this failure include not enough nursing staff, lack of education plan at the discharge time and ineffective supervision. Using this checklist is beneficial for better education outcomes. The findings recommend nurses to evaluate the economic, safety, and psychosocial situation of the patients as they may prevent the patient's and their family's ability to follow the discharge plans.
\end{abstract}

Keywords: Coronary Angiography, Patient Education, Discharge, Nursing Audit

\section{Background}

Cardiovascular disease is the leading cause of death for both males and females of all races and ethnicities. About 610000 people die of cardiovascular disease in the United States every year, which is one in every four deaths (1).

Coronary artery disease (CAD) is the most common type of cardiovascular disease and the standard diagnostic procedure for assessing CAD is coronary angiography (CAG), which is an invasive method $(2,3)$. Diagnostic CAG has been developed in to a safe process with a low complication rate. Nevertheless, some possible endangering complications are accompanied by CAG. Death, cerebrovascular accident, myocardial infarction, bleeding, hematoma, arterial thrombosis, vessel injury, infection and re-hospitalization are the most common CAG complications $(4,5)$.
Heart failure is the most frequent cause of readmission to the hospital and one-half of the cases experience readmission within six months due to exacerbation of symptoms and this happens as a result of inadequate knowledge (6). These frequent re-admissions can in turn impose burdens on the society from several perspectives (7). Therefore, to prevent re-hospitalization, promote their quality of life, and develop prognosis, improvement of strategies is essential (8). Among different therapeutic approaches, behavioral changes have proved to be safe and effective.

Interventions such as education have been reported to be effective for patients with CAD. As a result, detecting the patient's priority, planning for sufficient education and easing the patient's participation in a cardiac recovery program are the most efficient ways for raising patients' self-care plan following the discharge (9). 
Closely related to this issue is standard discharge education. It is widely accepted that the standard is a validated guide for practitioners and has the following features: they are validated, by an authorized expert, and connected to the people influenced on them. Since the standards are used as instruments for measurement purposes, they should be impartial, quantifiable and feasible (10). Therefore, standardizing discharge education for patients with cardiovascular disease can lead to a decrease in readmissions and an increase in patient satisfaction from nursing care (11).

In the review of the literature, the researchers did not find any discharge education for CAG patients' studies. Patient education is the one of the important duties of nurses to achieve the desired therapeutic outcomes at the hospitals. The nurses should perform healthcare practices with reference to a wide range of standards. Because nurses play a significant role in patient education, quality of their practice is critical for the recovery of CAG patients. To the best of our knowledge, this study was the first study about discharge education for CAG patients in Iran.

It is certain that a discharge program for CAG patients should contain self-care information about medication, nutrition, physical activity, weight control and selfmanagement (12). Besides, an appropriate plan for discharge should focus on education about healthy life style and disease management, which improves prognosis and reduces costs, because treatment adherence can lead to decreasing re-hospitalization rates (13).

\section{Objectives}

The present study was conducted to audit discharge education for CAG patients.

\section{Methods}

This study was a descriptive research. In total, 387 patients hospitalized at the cardiovascular clinic of three selected hospitals in Tehran, between May and September 2014 for CAG, were included in the current descriptive study.

Participants' selection was based on event sampling. The sample size was decided to be in proportion to the total CAG patients' admission per month.

Ethical agreement and approvals were received from the research and ethics committee of Shahid Beheshti Medical university (SBMU), Tehran, Iran (IRB number = 93-1-8613438). Informed permissions were obtained from all the participants, who took part in the study. Also, hospital managers were assured the hospital would remain anonymous.
A demographic questionnaire and checklist of discharge education for CAG patients were used in this study.

The demographic questionnaire was used to collect patients' demographic information and professional characteristics of the participating nurses.

Checklist of discharge education for CAG patients (CDECP): This observational measurement tool is a researcher-designed checklist arranged according to the literature and related guidelines. The CDECP is comprised of 35 items and six dimensions that assess discharge education related to CAG patients. The six dimensions of the CDECP are Nurse-patient communication (nine items), CAG wound care education (five items), bleeding control education (two items), drugs usage education (three items), emergency events education (eight items), and changing life style education (eight items). Comparing measures against the standards was evaluated as $0-33 \%$ $=$ weak level of education, $34 \%-66 \%=$ moderate level of education, and $67 \%-100 \%=$ high level of education.

The reliability and validity of the CDECP were approved by content validity and inter-rater reliability. Content validity was approved by ten experts (specialists in the cardiac nursing, assistant professors of nursing management and cardiologists). As such, the CVI for all the items was above 0.75 except one item that did not meet this criterion and so was omitted. The reliability of the CDECP was assessed through calculating inter-rater coefficient. For this purpose, two raters observed 10 discharge educations and completed the checklist. The inter-rater coefficient was $97 \%$, which is an acceptable index.

Mentioning the names of the hospitals separately in the results was avoided for the sake of research ethics. The researchers started data collection primarily by obtaining necessary permissions from Shahid Beheshti University of Medical Sciences and Ethics Committee, as well as the administrators of the hospitals. Then, one of researchers referred to the study setting each day in the morning, evening and night working shifts and performed structured observations of discharge patient educations by using the checklist of discharge education for CAG patients.

All data were expressed as means \pm standard deviation (SD), and analyzed by the SPSS 20 software (SPSS Inc., Chicago, IL, USA). We analyzed the data of the subjects, who completed the entire study. Initially, the total score of each observation was calculated and then the scores were changed to percentage values, i.e. a 0 - 100 scale. Finally, 0 - 100 scores were divided to three groups of $0-33$ (poor education), 34 - 67 (moderate education), and 68 - 100 (optimum education). 


\section{Results}

Out of 387 CAG patients, $50.4 \%$ were males and $49.6 \%$ females. The mean age of the patients was $60.25 \pm 12.26$ years. Overall, $79.1 \%$ of the patients were married, $29.7 \%$ were illiterate, and $62.8 \%$ reported no CAG admission before.

Furthermore, $95.2 \%$ of the observed nurses were graduates of bachelor of science (BSc) in nursing. In addition, their mean professional experience in nursing (year) was $11.26 \pm 5.7$ and mean job experiment in CAG ward (year) was $3.20 \pm 1.78$.

Comparison of the observed discharge education against the standards revealed a comparison measure of $24 \%$ for all dimensions and the maximum measure was found in CAG wound care education dimension (48.3\%). Comparison measures for other dimensions were $37.3 \%$ for bleeding control education, 30.6\% for nurse-patient communication, $22.9 \%$ for changing life style education, 9.3\% for emergency events education, and finally $2.15 \%$ for drugs usage education (Table 1).

Moreover, no significant difference was found among the comparison measures of the three hospitals against the standards of discharge educations for CAG patients (Table 2).

\section{Discussion}

The results of the present study showed that no adequate discharge education is provided for CAG patients and this is while these patients, due to the invasiveness of the procedure, are at a high risk of vascular complications. Hence, providing sufficient education and information for these patients seems vital.

Several previous observational studies have also demonstrated our findings. For example, in a retrospective auditing study about nursing care of heart defect patients, it was shown that there exists a low level of nursing care (15.9\%), similar to what was found for discharge education (14).

Another survey reported interesting estimates about discharge auditing. They found that nurses often had no responsibility regarding patient discharge coordination, and other unprofessional people were involved in this process (15). In addition, in another study, it was found that most discharge summary reports were not in accordance with the standards (16). Furthermore, findings reported by Makaryus and Friedman (2005) displayed that less than half of the patients that inquired during the discharge did not have adequate knowledge about the names of their drugs, purpose of their medications, and their common side effects (17).
In contrast, findings in some studies were different from those of the present study. For example, a study about professional nurses' perception about patient education revealed that educating patients was the priority of nursing care among $92 \%$ of the observed nurses. These differences reflect the existing gap between nurses' perception and practice (18). Another study examined patients' understanding of the discharge treatment plan. The researchers determined patients' awareness and compliance with regards to their treatment plan, 7 to 14 days after hospitalization (19).

Based on the findings, $73 \%$ of the observed patients were not only able to list the names of all their medications but they could also describe both the purpose and common side effects of their medications. The satisfactory findings in these studies can be due to the long length of hospitalization of the patients, during which nurse-patient communication becomes more and patients ask limited questions on topics like medications, yet other important topics are neglected by nurses. At any rate, lack of awareness about and abilities for both home care and lifestyle modification after discharge can slow down the process of getting well by bringing about physical and mental stress on the patients (20). Inclusive discharge education led to better health consequences such as better quality of life and not being re-hospitalized (21).

Also, various studies declared that discharge education seems necessary and useful to reduce anxiety and depression, re-hospitalizations, and non-compliance to medication, to improve survival and quality of life, and to empower patients to preserve the ability to control their lives $(22,23)$. In this regard, education is essential for patients to recognize the process of the disease, because increasing patients' information can result in development of selfcare and decrease in hospitalization rates (24). Additionally, CAD patients should elevate their exercise gradually, improve usage of their medication, promote control of signs and symptoms indicating the worsening of their illness, and continue their follow-up program carefully (25).

Also, nurse-patient communication increases faith in the profession and reassures obedience from the discharge plan in patients (26). Moreover, provision of a one-hour educational session by nurse educators at the time of hospital discharge is reported to improve patient information compared with the standard discharge process. In fact, inadequacy in CAG patients' knowledge about discharge plan is strongly correlated with higher risk of rehospitalization (27).

Presence of the researchers in the study environment, i.e. cardiac care wards, for observing and auditing purposes might have influenced the caregivers' performance. To resolve this limitation, the purposes of the study and the 
Table 1. Measure of Standard Execution in Each Sub-Domain According to The Checklist of Discharge Education for Coronary Angiography (CAG) Patients (CDECP)

\begin{tabular}{|c|c|c|}
\hline Sub-domain & Performed, No. (\%) & Not performed, No. (\%) \\
\hline Nurse-patient communication ( 9 items) & 1068 (30.66), Poor education & $2415(69.34)$ \\
\hline CAG wound care education (5 items) & 936 (48.38), Moderate education & $999(51.62)$ \\
\hline Bleeding control education ( 2 items) & 289 (37.34), Moderate education & $485(62.66)$ \\
\hline Drugs usage education (3 items) & 25 (2.15), Poor education & $1136(97.85)$ \\
\hline Emergency event education (8 items) & 288 (9.3), Poor education & $2808(90.7)$ \\
\hline Changing life style education ( 8 items) & 710 (22.93), Poor education & $2386(77.07)$ \\
\hline
\end{tabular}

Table 2. Comparing Measure of Discharge Education for Coronary Angiography (CAG) Patients Against the Standards at Selected Hospitals

\begin{tabular}{|c|c|c|c|c|c|}
\hline Hospitals & Standard assignment $^{\mathrm{a}}$ & Present condition & Accordance measure, $\mathrm{Y} / \mathrm{X}$ & Mean \pm SD & CI \\
\hline A & $161 \times 35=5635, x_{1}=5635$ & $\mathrm{Y}_{1}=1441$ & 0.25 & $8.95 \pm 2.35$ & $8.58-9.31$ \\
\hline B & $129 \times 35=4515, x_{2}=4515$ & $Y_{2}=1113$ & 0.25 & $8.62 \pm 2.84$ & $8.13-9.12$ \\
\hline C & $97 \times 35=3395, x_{3}=3395$ & $Y_{3}=764$ & 0.22 & $7.88 \pm 1.92$ & $7.49-8.26$ \\
\hline Total & $387 \times 35=13545 x_{T}=13545$ & $Y_{4}=3318$ & 0.24 & $8.57 \pm 2.46$ & $8.33-8.82$ \\
\hline
\end{tabular}

${ }^{\mathrm{a}}$ Standard assignment $=$ number of observed nursing education $\times$ Total items of check list.

reasons for their continuous presence in the environment were explained to the caregivers.

\subsection{Conclusion}

The results of the present study suggest that CAG patients receive inappropriate discharge education. According to the findings, the current discharge education seems to be ineffective in comparison with the standards from various aspects. Consequently, it seems that, hospital discharge plans carried out by nurses should seriously be re-considered. In addition, better interventions such as open and trustworthy communication may support both discharge education and patient self-care. Furthermore, nurses should begin the discharge program at the patients' admission for identifying their personal and family history as well as psychological, social, and physiological characteristics to find re-hospitalization variables related to the admission and discharge, which leads to promotion of an individualized program.

To make the findings of the present study more robust, supplementary research is necessary for addressing the advancement and execution of education during discharge programs and also the impact of such programs on CAG patients, confirmation of patients' information, families' support of the discharge programs, and the development of educational equipment and supplies to educate patients. A hospital discharge planned by nurses should be individualized. Lack of communication among nurses and caregivers delays the care plan and thus must be overcome. For better education outcomes, nurses should also evaluate the economic, safety, and psychosocial requirements of patients that may hamper the patient's and family's ability to follow the discharge plan. Open and trustworthy communication supports care, promotes education, and organizes a discharge plan.

The present study dealt solely with the accordance of discharge education for CAG patients with the standards. Consequently, further studies are needed for uncovering the reasons behind poor compliance with the standards. Developing and implementing discharge education guidelines and supervising their accurate implementation are among the first steps for the provision of quality and standard discharge education for CAG patients.

\section{Acknowledgments}

This study was part of a Masters thesis approved by Shahid Beheshti University of Medical Sciences. The funding of this research was provided by the Shahid Beheshti University of Medical Sciences, Tehran, Iran. The authors appreciate all the authorities of Shahid Beheshti University of Medical Sciences for financially supporting this research.

\section{Footnotes}

Authors' Contribution: Study design: Fatemeh Davoodi and Foroozan Atashzadeh-Shoorideh; data collection and analysis: Fatemeh Davoodi, Abolfazl Payandeh and Foroozan Atashzadeh-Shoorideh; counseling: Mahnaz Ilkhani, Abolfazl Payandeh and Hossein Shiri; manuscript preparation and edit: Foroozan Atashzadeh-Shoorideh, Sara Mahmoody, Marjan Mohamadi and Hadi Azimi. 
Conflicts of Interest: The authors declare no conflicts of interest.

Funding/Support: The funding for this research was provided by the Shahid Beheshti University of Medical Sciences, Tehran, Iran.

\section{References}

1. CDC . Underlying cause of death 1999-2013 US: Center of Disease Control and Prevention; 2013. Available from: http://www.cdc.gov/ dhdsp/data_statistics/fact_sheets/fs_heart_disease.htm.

2. Smeltzer SC, Bare BG, Hinkl JL, Cheever KH. Brunner and Suddarth's textbook of medical surgical nursing. 12 ed. Lippincott: Williams and Wilkins; 2014.

3. Buzatto LL, Zanei SS. Patients' anxiety before cardiac catheterization. Einstein (Sao Paulo). 2010;8(4):483-7. doi: 10.1590/S167945082010RW1517. [PubMed: 26760335].

4. Nguyen T, Dayi H, Shaoliang C, Kim M, Saito S, Grines C. Practical handbook of advanced interventional cardiology. 4 ed. Hong Kong: Wileyblackwell; 2013.

5. Howlett JG. Palliative care in heart failure: addressing the largest care gap. Curr Opin Cardiol. 2011;26(2):144-8. doi: 10.1097/HCO.ob013e3283437468. [PubMed: 21252651].

6. Giamouzis G, Kalogeropoulos A, Georgiopoulou V, Laskar S, Smith AL, Dunbar S, et al. Hospitalization epidemic in patients with heart failure: risk factors, risk prediction, knowledge gaps, and future directions. J Card Fail. 2011;17(1):54-75. doi: 10.1016/j.cardfail.2010.08.010. [PubMed: 21187265].

7. Thomas JR, Clark AM. Women with heart failure are at high psychosocial risk: a systematic review of how sex and gender influence heart failure self-care. Cardiol Res Pract. 2011;2011:918973. doi: 10.4061/2011/918973. [PubMed: 21403845].

8. Gonseth J, Guallar-Castillon P, Banegas JR, Rodriguez-Artalejo F. The effectiveness of disease management programmers in reducing hospital re-admission in older patients with heart failure: a systematic review and meta-analysis of published reports. Eur Heart J. 2004;25(18):1570-95.

9. Saffi MA, Polanczyk CA, Rabelo-Silva ER. Lifestyle interventions reduce cardiovascular risk in patients with coronary artery disease: a randomized clinical trial. Eur J Cardiovasc Nurs. 2014;13(5):436-43. doi: 10.1177/1474515113505396. [PubMed: 24021286].

10. Marquis B, Huston C. Leadership roles and management functions in nursing,Theory and Application. Philadelphia: Lippincott Williams and Wilkins; 2012.

11. Perloe M, Rask K, Keberly ML. Standardizing the hospital discharge process for patients with heart failure to improve the transition and lower 30 day readmissions. Laguna Niguel: The Remington Report; 2011.

12. AHA . New program extends health education, support for heart patient failure after hospital discharge, program news. US, Dallas: American Heart Association; 2015.

13. Polster D. Preventing readmissions with discharge education. Nurs Manage. 2015;46(10):30-7. doi: 10.1097/01.NUMA.0000471590.62056.77. [PubMed: 26359555].
14. Ehrenberg A, Ehnfors M, Ekman I. Older patients with chronic heart failure within Swedish community health care: a record review of nursing assessments and interventions. J Clin Nurs. 2004;13(1):90-6. [PubMed: 14687298].

15. Watts R, Pierson J, Gardner H. Co-ordination of the discharge planning process in critical care. J Clin Nurs. 2007;16(1):194-202. doi: 10.1111/j.1365-2702.2005.01439.x. [PubMed: 17181682].

16. Dinescu A, Fernandez H, Ross JS, Karani R. Audit and feedback: an intervention to improve discharge summary completion. J Hosp Med. 2011;6(1):28-32. doi: 10.1002/jhm.831. [PubMed: 21241038].

17. Makaryus AN, Friedman EA. Patients' understanding of their treatment plans and diagnosis at discharge. Mayo Clin Proc. 2005;80(8):991-4. doi: 10.4065/80.8.991. [PubMed: 16092576].

18. Marcum J, Ridenour M, Shaff G, Hammons M, Taylor M. A study of professional nurses' perceptions of patient education.J Contin Educ Nurs. 2002;33(3):112-8. [PubMed: 12046712].

19. Kerzman H, Baron-Epel O, Toren O. What do discharged patients know about their medication?. Patient Educ Couns. 2005;56(3):276-82. doi: 10.1016/j.pec.2004.02.019.

20. Ivarsson B, Larsson S, Luhrs C, Sjoberg T. Extended written preoperative information about possible complications at cardiac surgery-do the patients want to know?. Eur J Cardiothorac Surg. 2005;28(3):407-14. doi: 10.1016/j.ejcts.2005.05.006. [PubMed: 16055340].

21. Yu J. Application of continuing care in patients with generalized anxiety disorder after discharge. Innov $J$ of Med and Health Sci. 2015;5(3):107-10.

22. Berben L, Bogert L, Leventhal ME, Fridlund B, Jaarsma T, Norekval TM, et al. Which interventions are used by health care professionals to enhance medication adherence in cardiovascular patients? A survey of current clinical practice. Eur J Cardiovasc Nurs. 2011;10(1):14-21. doi: 10.1016/j.ejcnurse.2010.10.004. [PubMed: 21095160].

23. Veronovici NR, Lasiuk GC, Rempel GR, Norris CM. Discharge education to promote self-management following cardiovascular surgery: an integrative review. Eur J Cardiovasc Nurs. 2014;13(1):22-31. doi: 10.1177/1474515113504863. [PubMed: 24042728].

24. Chrvala CA, Sherr D, Lipman RD. Diabetes self-management education for adults with type 2 diabetes mellitus: A systematic review of the effect on glycemic control. Patient Educ Couns. 2016;99(6):926-43. doi: 10.1016/j.pec.2015.11.003. [PubMed: 26658704].

25. Negarandeh R, Nayeri ND, Shirani F, Janani L. The impact of discharge plan upon re-admission, satisfaction with nursing care and the ability to self-care for coronary artery bypass graft surgery patients. Eur J Cardiovasc Nurs. 2012;11(4):460-5. doi:10.1016/j.ejcnurse.2011.05.001. [PubMed: 21640653].

26. Murante AM, Vainieri M, Rojas D, Nuti S. Does feedback influence patient - professional communication? Empirical evidence from Italy. Health Policy. 2014;116(2-3):273-80. doi: 10.1016/j.healthpol.2014.02.001. [PubMed: 24630781].

27. Kommuri NV, Damodaran A, Koelling TM. Discharge education improves knowledge and outcomes in heart failure patients. $J$ American College of Cardiol. 2010;55(10):A29.E280. doi: 10.1016/s07351097(10)60281-1. 